\title{
TRATAMIENTO DE LA PREECLAMPSIA-ECLAMPSIA (*)
}

\author{
Doctor Fernando Sánchez Torres
}

(Del Servicio de Toxemias del Instituto Materno-Infantil

"Concepción Villaveces de Acosta")

\section{Introducución}

Para poder emprender racionaimente un tratamiento dirigido a detener y vencer cualquier proceso patológico, es condición primera identificar con precisión la causa o el agente que lo origina, junto de un conocimiento amplio de las alteraciones que éste ocasiona en el organismo atacado. Entre el sinnúmero de entidades que constituyen la patología humana pocos son los estados morbosos que aún permanecen ignorados en su etiología verdadera. De este grupo reducido forma parte la llamada "Toxemia del último trimestre del embarazo" o "Preeclampisia_eclampsia", cuyo factor o factores causales permanecen todavía en el campo de las probabilidades.

Sin embargo, se puede hoy afirmar que la preeclampsiaeclampsia es evitable, y que una vez instaurada es controlable gracias a la ilustración que de su fisiopatología se ha logrado. El hecho claro de que sin placenta no hay toxemia, ha inducido a todos los investigadores a querer encontrar en ella la fuente original del proceso. De ser así, ahí tendría su punto de partida el elemento incógnito que ocasiona el cataclismo, la disrritmia global de un organismo materno que hasta entonces venía acomodándose de manera aparente, o completamente normal, a la sobrecarga impuesta por la gravidez, pero que, transpasado el umbral del tercer trimestre, se descquilibra silenciosamente con una sucesión progresiva de hechos que van a desembocar en la tempestad convulsiva.

La concepción fisiopatológica de la toxemia tard'a del embarazo pudiéramos resumirla así:

(*) Presentado al IV Congreso Colombiano de Obstetricia y Grinecología efectuado en Barranquilla del 29 de noviembre al 3 de diciembre de 1959. 


\section{Fisiopatología}

Sometido desde meses atrás el organismo femenino a una lenta reacción inespecífica de defensa o de adaptación general al stresis producido por el embarazo, que ha venido obrando como un alarmógeno (14), ese organismo claudica en un momento dado, bien sea por influencias síquicas o por intensificación brusca del alarmógeno_hiperfunción placentaria (3), constituyéndose una verdadera enfermedad de adaptación.

El sistema diencéfalo-hipófiso-adrenal estimulado (14) origina el cortejo de manifestaciones: desequilibrio de los esteroides específicos con aumento de los mineralocorticoides, lo que acarrea alteración del metabolismo hidromineral por acción sobre el túbulo renal proximal con la consiguiente absorción de cloro y sodio y eliminación de potasio (3). Este desequilibrio electrolítico, junto con el aumento de gonadotropinas coriónicas, eleva la con. centración de hormonas antidiurética, vasopresora y ocitócica al excitar la zona osmorreceptora de la hipófisis posterior $(3,21)$.

Tengamos ien cuenta que ya ha aparecido como personaje central el ión sodio que habrá de comportarse como la verdadera toxina de la preeclampsia_eclampsia.

Siendo el epitelio del túbulo renal una de las más importantes membranas relacionadas con cl equilibrio electrolitico, la más mínima alteración de su función normal se reflejará en trastornos del volumen y composición de los fluídoミ (17). Como la reten. ción de sodio siempre se acompaña del agua suficiente para mantener su isotonicidad, el edema, oculto o aparente, inicia el fatal encharcamiento de los espacios extravasculares, disimulado por una cifra alta del hematocrito y normal o baja concentración de sodio en el suero, al paso que en el fluído del edema se halla aumentado dicho catión, lo que no sucede er el edema de pacientes embarazadas no toxémicas (24).

El ediema, o sea la retención hidrosalina, es, pues, el primer signo en aparecer (17). El vasoespasmo ocasionado por factores neurogénicos y humorales (11) no tarda en manifestarse reforzado por un completo círculo vicioso. El riñón afectado ya por la gran presión intra-abdominal que ocasiona la presencia del útero yrávido; atacado directamente en su epitelio tubular por la adiuretina y las hormonas esteroides; sus células henchidas de agua; estrechadas sus arteriolas con la consiguiente anoxia y deficiente 
riago, contribuye a la catástrofe, aumentando la permeabilidad de sus capilares glomerulares eliminando proteínas y produciendo sustancias vaso_activas (reninas tonógenas) que van a sumar su acción hiperterisora a la que ocasiona la vasopresina y la adrenalina.

EI útero con deficiente aporte sangu'neo (18), estimulado ¿xtemporáneamente por la ocitocina (demostrable por la contractilidad elevada) (1) tiende a expulsar con precocidad su inmaciuro contenido, al que se había ya proyectado la conmoción materna por una placenta críticamente irrigada y extensamente infartada (13).

El desequilibrio electrolítico, el edema progresivo y las alteraciones de 02 y $\mathbf{C} 02$ trastorna la actividad eléctrica normal de la corteza $(11,21)$, irrumpiendo ientonces, como última etapa del proceso, la estridente eclampsia que es manifestación de la profunda perturbación del cerebro.

\section{Tratamiento de la preeciampsia}

Comprendida así la fisiopatología, el tratamiento de la preeclampsia tendrá que ir encaminado en primer término a desalojar del organismo afectado los dos elementos que en suma originan todas las alteraciones: Eodio y agua. La prueba fehaciente de que es así lo demuestra el hecho de que aquellas mujeres embarazadas a las que se les ha vigilado con severidad su metabo. lismo hidrosalino, difícilmente formarán parte del grupo de pacientes con toxemia. Plena razón asiste a quien afirma que la preeclampsia no es más que un problema de equilibrio hídrico (17), y a lo que se pudiera añadir que es sólo una intoxicación hidrosalina cuyas causas desencadenantes ignoramos.

Conseguir que el organismo que vamos a tratar se coloque en condiciones similares a las de una gestante normal, ha de ser nuestro empeño principal. Si promovemos la movilización de los fluídos estancados para que sean excretados, el cortejo de manifestaciones que acompaña a la toxemia tendrá que ahuyentarse (17). No así, y esto es lógico, las profundas alteraciones placentarias, causa o consecuencia de la toxemia, cuya presencia constituirá una permanente amenaza a la vitalidad fetal (13).

Es conveniente anotar aquí que nosotros hacemos caso omiso de la intensidad de los signos edema, hipertensión y proteinuria para calificar la gravedad de la preeclampsia. Consideramos que 
toda paciente con toxemia podrá desembocar en eclampsia, tar día o precozmente, según la mayor o menor capacidad de adaptación al desequilibrio instaurado. Pacientes hay que soportan sin manifestación convulsiva una tensión elevada y grandes edemas, y otras que con edemas discretos y ligera hipertensión hacen eclampsia sorpresiva. Interesa, sí, catalogarla como toxémicas puras o con enfermedad hipertensiva crónica asociada, ya que esta última contingencia nos autoriza a hablar de preeclampsia grave debido al mal pronóstico que siempre tienen la madre y, especialmente, el feto.

Las primeras medidas que tomamos en caso de preeclampsia son iel reposo, que reduce las exigencias que el organismo impone al sistema circulatorio, y la natural restricción de cloruro de sodio y agua. De 500 miligramos a 1 gramo diario de sal con la alimentación serán permitidos. Se podrán ingerir de 700 c. c. a 1 litro de agua en las 24 horas. El tratamiento de fondo lo hacemos con diuréticos, siendo el preferido la clorotiazida, ya que su actividad salurética solo es comparable a la de los diuréticos mercuriales sin los inconvenientes de éstos. Su mecanismo de acción es inhibir la reabsorción tubular de sodio promoviendo una diuresis in. tensa, afectiva. Tiene la ventaja sobre los inhibidores de la anhicrasa carbónica de no ocasionar resistencia cuando se administra durante tiempo prolongado (15). La dosificación y duración se deben ajustar a la necesidad individual. En los casos de edema intenso suministramos un gramo en 24 horas y 500 miligramos en ios de edema moderado.

Hasta aquí hemos librado al organismo del exceso de agua y sodio, quedándonos aún por contrarrestar el factor neurogénico y la producción de substancias que influyen en la retención hidrosalina y en la hipertensión, es decir, la vasopresión y la adiuretina. Para conseguir tal efecto, prescribimos barbitúricos de acción prolongada tipo fenobarbital sódico en dosis de 100 miligramos cada 8 horas, que producen benéfica sedación a la vez que frenan suavemente, por influencia diencefálica (16), la excesiva producción dé las llamadas hormonas post-hipofisiarias.

Cuando los edemas hayan desaparecido y la cifra tensional alcance niveles normales, la paciente debe seguir un régimen dietético equilibrado en calidad y cantidad, sin prohibirle la ingestión de sodio. No olvidemos que el riñón excreta con mayor actividad el ión potasio cuando hay deficiencia de sodio (25). 
La clorotiazida se prescribe luégo en dosis de 500 miligramos tres veces a la semana y los barbitúricos 100 miligramos una 0 dos veces al día, hasta el final del embarazo o hasta el momento en que creamos oportuna la inducción del parto. Durante este lapso por lo menos dos veces a la semana se controlará la tensión arterial, el peso y se investigará la protcinuria, Cualquier agravación nos hará sospechar que la paciente no sigue exactamente nuestras recomendaciones o que la terapéutica debe intensificarse.

Discrepamos de la tendencia actual de emplear sistemáticamente substancias hipotensoras específicas en todas las formas de toxemia gravídica. Solo las utilizamos en los casos de afección hipertensiva crónica, con o sin toxemia sobreagregada. Bien sa. bemos de la acción hipotensora ideal de la clorotiazida, que sin ser directa va a obrar disminuyendo la resistencia intrínseca de los vasos y sobre todo al mejorar la función renal (15). Comprobado está que mientras haya retención de agua y sodio el objetivo perseguido con las substancias hipotensoras específicas puede fracasar (25). El no usar vasodepresores rutinariamente nos ha servido para diferenciar en muchas ocasiones la toxemia pura de la hipertensión crónica y hemos evitado que con su empleo pase desapercibida una agravación de la preeclampsia. En el primer caso, por más alta que sea la cifra tensional, siempre volverá a niveles normales con el tratamiento expuesto atrás. Si tal cosa no sucede podemos afirmar que hay un componente hipertensivo crónica. Hacemos uso entonces de un antihipertensor ospecífico.

\section{Tratamiento de la eclampsia}

No es nuestra intención divulgar un tipo estandard de tratamiento médico para la eclampsia, es decir, prefijar una serie de medicamentos, indicar sus dosis y vías de administración y recomendarlos de manera general para toda paciente con toxemia convulsiva. Apenas queremos señalar normas básicas, elementales, pero sujetas a modificación. No nos equivocamos al decir que para cada caso el tratamiento en principio es el mismo, pero sometido a variación según las circunstancias. El que desconozca este hecho desconoce el tratamiento médico razonable. Creemos que aquí radican los fracasos del tratamiento conservador en la eclampsia, que se moteja entonces "incontrolable" para justificar la conducta quirúrgica. 
La terapéutica que vamos a exponer es la base del tratamiento adoptado en el Servicio de Toxemias del Instituto MaternoInfantil "Concepción Villaveces de Acosta" desde hace cuatro meses con resultados verdaderamente sorprendentes. De $27 \mathrm{ca-}$ sos de eclampsia no hemos tenido ninguna defunción materna y sólo dos muertes fetales: una de un feto de 800 gramos y otra consecutiva a un abruptio placentae. Si comparamos este resultado con los obtenidos cuando seguíamos otras formas de tratamiento, tenemos que aceptar la bondad del actual.

De 37 eclampsias tratadas de julio a diciembre de 1958 con el tan en boga "cocktail lítico" fallecieron 6 mujeres y 10 niños y 4 pacientes hicieron posteriormente cuadros de sicosis puerperal.

De enero de 1956 a septiembre de 1957 fueron tratados 145 casos de eclampsia en la siguiente forma y con los siguientes resultados:

Con Dextrosa_Largactil intravenoso 49 casos, de los cuales fallecieron 5 .

Con Dextrosa-Largactil intravenosos y Sulfato de Magnesia intrarraquídeo, 45 eclampsias con 5 muertes.

Con Pentotal-Dextrosa intravenosos, 24 casos con 2 fallecimientos.

Con tratamientos combinados, 27 eclampsias con 4 muertes maternas (22).

\section{Normas y cuidados generales}

Un ambiente tranquilo, lejos de todo estimulo exterior, es el medio ideal para mantener a la paciente con eclampsia. No es recomendable fijar sus miembros con ligaduras temiendo que caiga de su lecho, lo cual se evita usando catre con barandillas. Sobra decir que en el instante del ataque se debe evitar la mordedura de la lengua introduciendo parte de una compresa entre la boca. De olvidarse tal precaución podrá presentarse una verdadera amputación o heridas que hagan necesaria su inmediata sutura. La oxigenación debe ser satisfactoria, manteniendo libres las vías respiratorias, llegando, $\mathrm{si}$ es el caso, a practicar una traqueotomía (23). La administración de oxigeno se hará durante los 30 minutos que siguen al ataque, por medio de tienda o sonda nasal.

Aconsejamos prescindir de cualquier examen clínico antes de aplicar la terapéutica “de ataque”. Hemos advertido que el estí- 
mulo que ocasionan el tomar la tensión arterial, el tacto vaginal o el cateterismo vesical son capaces, por sí solos, dè desencadenar nuevas convulsiones. Toda paciente que presente ataques convulsivos y quie tenga más de cinco meses de embarazo debe conside. rarse como una eclámptica hasta que se demuestre lo contrario. No debemos temer si nos equivocamos de diagnóstico, ya que los medicamentos que utilizamos tienen empleo en cualquier entidad convulsivante.

Una vez que la medicación sedante haya hecho su mayor efecto procedemos a la exploración clínica y colocamos una sonda vesical a permanencia.

\section{Tratamiento medicamentoso}

Existiendo cronológicamente en relación con el embarazo 1res tipos de eclampsia -anteparto, intraparto y post-parto-, es natural que el tratamiento medicamentoso varie en cada caso. Cuando han transcurrido más de 24 horas sin haberse repetido las convulsiones, se considera que la paciente ha entrado en "eclampsia intercurrente" (23).

Nos hemos impuesto como lema en la terapéutica de la eclamp. sia usar lo mínimo que resulte eficaz. Por eso sólo empleamos tres substancias medicamentosas en la mínima dosis suficiente para detener las crisis convulsivas sin que causen daño a la madre o al feto.

Esos tres medicamentos son el Fenobarbital sódico, el Sulfato de Magnesia y la Acepromazina.

El Fenobarbital sódico, barbitúrico de acción prolongada, ha sido considerado siempre como el anticonvultivante por excelencia. Su acción principal es deprimir el sistema nervioso central (corteza cerebral y porción talámica del diencéfalo) (9). Administrado por vía endovenosa produce hipotensión arterial pasajera y si se aplica demasiado rápido puede causar depresión respiratoria. No afecta en nada la función renal y sólo lo hace cuando administrado en grandes dosis lleva a un prolongado período de depresión profunda (12).

El uiso de la morfina o de los barbitúricos ha tenido preferen. cia según los diferentes autores. Dieckmann recomienda $11 / 2$ a 2 centigramos de morfina para dominar las convulsiones sin pasar de 12 centigramos en 24 horas, en tanto que Assali aconseja 3 gramos de barbitúricos intramuscular o intravenosamente, repitién- 
dose si es necesario (2). León no aplica barbitúricos porque, según él, "disminuyen la circulación sanguínea cerebral y deprimen la respiración" (14), mientras Tatum los prescribe de acción corta o ultracorta en dosis de 200 a 500 miligramos por vía endovenosa, seguido de 200 miligramos de Fenobarbital intramuscu. $\operatorname{iar}(23)$.

Nosotros usamos como dosis de ataque 200 miligramos de Fenobarbital sódico por vía endovenosa, es decir, apenas la dosis hipnótica media para un adulto (12), pero potencializada gracias al empleo simultáneo de Acepromazina (7). No usamos la morfina, ya que su potencia anticonvulsiva es relativamente escasa, evidenciándose depresión de la corteza motora luego de aplicar grandes dosis (12).

El Sulfato de Magnesia parenteral disminuye la excitabilidad nerviosa, deprime el sistema nervioso central y tiene ligera acción diurética. Su gran actividad es ocasionar dilatación artieriolar seguida de mayor oxigenación de los tejidos e hipotensión arterial pasajera. Muchos lo consideran como un agente anticonvul. tivante de primer orden. Se ha usado en dosis parenteral hasta de 30 gramos en 24 horaฐ. La solución indicada para la vía endovenosa es al 10 o $20 \%$ y al $50 \%$ para la intramuscular. Esta via establece una acción anticonvulsivante más uniforme que la producida por aquélla $(2,4,14,23)$. Sin embargo, nosotros preferimos la intravenosa, pues lo que buscamos es detener prontamente ias convulsiones. Aplicamos 15 c. c. de solución al 15\% muy len¿amente, repitiendo la misma cantidad media o una hora después. La dosis total de sulfato de magnesia que empleamos es de 4 a $4 \frac{1}{2}$ gramos.

El advenimiento de los derivados de la Fenotiazina ha abierto un gran paréntesis de optimismo al tratarniento médico de la eclampsia debido a sus múltiples efectos beneficiosos: frenan o paralizan el sistema nervioso central (acción neuropléjica), frenan o paralizan los ganglios basales (acción gangliopléjica), producen caída de la tensión nerviosa o neurovegetativa (acción neuioléptica) y hacen caer la tensión sicológica (acción sicoléptica) (5).

La forma más común como se han venido usando las Fenotiazinas en la eclampsia es en goteo con mezclas en las que predomina la Clorpromazina o Largactil, practicando con ellas una invernación de tipo químico. Los resultados con tal forma de tratamiento no siempre se acompañan de un éxito completo. Noso- 
tros de 37 eclámpticas que tratamos con cockteles líticos fracasamos en un buen número de casos y, lo más lamentable, contabilizamos seis muertes maternas. Arcadio Sánchez López, del Insti_ iuto Provincial de Obstetricia y Ginecologia de Madrid, en su primer informe sobre lo que él llama "Hibernación curativa" de la eclampsia con neuropléjicos presenta solo 5 casos: 3 del puerpe. rio resueltos favorablemente y 2 del preparto, uno de los cuales termina en cesárea por "sufrimiento fetal, hipertonía uterina y ausencia de toda modificación pasiva cervical" (21). J. Ruzicska y B. Zsolnai informan en 1957 haber tratado 8 eclampsias consiguiendo hacer cesar en 5 las convulsiones, teniendo que terminar el parto en 3 con fórceps por "insuficiencia contráctil". En los 3 casos restantes no lograron dominar los ataques y tuvieron que apelar a la cesárea (20). Santiago Dexeus a fines de 1958 considera el tratamiento de las gestosis con Fenotiazinas como moneda casi habitual, pero deja escuchar estas palabras llenas de pesimismo: "Resulta difícil juzgar certeramente cuál es el momento en que tenemos que dejar este tratamiento que podemos llamar médico para, frente al fracaso del mismo, lanzarnos en brazos de una solución más activa, generalmente la operación cesárea" (8).

Considerando los neuropléjicos como valioso complemento en el tratamiento de la eclampsia resolvimos abandonar su empleo abusivo en mezclas o cockteles para utilizarlos en la forma y do_ sis exactamente necesarias. Escogimos de todos el de más potente acción y menor toxicidad, que es el denominado comúnmente Acepromazina o Acetil 3 (Dimetilamino 3-propil) 10 Fenotiazina que comparado con la Clorpromazina es dos veces más activo y mucho menos tóxico. La dosis media recomendada en 24 horas para el adulto es de 30 a 50 miligramos (6). Nosotros utilizamos como dosis total en el tratamiento de la eclampsia de 6 a 8 miligramos por vía intramuscular, repartidos en dos dosis.

Con 200 miligramos de Fenobarbital, con 4 gramos de sulfato de magnesia y con 6 miligramos de Acepromazina hemos venido desde hace cuatro meses tratando la eclampsia con un re. sultado que modestamente consideramos alentador, ya que en ninguna ocasión hemos fracasado. Creemos que el éxito de nuestro tratamiento estriba en que conseguimos dominar rápidamente la fase convulsiva de la toxemia gravídica al encontrar la dosis mínima efectiva de tres suibstancias medicamentosas sin causar nin. gún daño a la madre o al feto. 


\section{Tratamiento deshidratante o desintoxicante}

Frenadas las convulsiones con la administración simultánea de Fenobarbital, sulfato de magnesia y acepromazina, podemos zecir que hasta aquí sólo hemos apaciguado el más escandaloso signo de la toxemia, quedándonos aún por combatir la verdadera causa que la ocasionó: la intoxicación hidrosalina.

Tenemos que creer firmemente que la acumulación de sodio $y$ agua en los espacios extravasculares, junto con el arterioloespasmo, debido a éste y otros mecanismos ya enumerados, causan todo el cataclismo en el organismo de la mujer afectada de toxemia. De no ser así, ¿por qué en todos los reportes de Anatomía Patológica de pacientes que fallecieron por eclampsia se lee ruti. nariamente: Riñón: hemorragias patequiales y edema de los capilares glomerulares. Cerebro: edema y hemorragias macro y microscópicas. Hígado: hemorragias periportales y edema intracelular. Pulmón: congestión y edema? (19).

Unánimemente los autores están de acuerdo en que es de fundamental importancia estimular la deshidratación. Desde la medicación purgante y la sangría muy usadas ęn el siglo pasado hasta la glucosa hipertónica preferida en la actualidad, siempre se ha buscado la manera de deshidratar de urgencia a la eclámptica. Dieckmann, citado por Juan León (14), inyecta hasta un litro de Dextrosa al $20 \%$ en un lapso de 30 a 50 minutos, repitiéndolo cada 6 a 8 horas. Botella Llusiá recomienda en su método o "Método de Madrid" aplicar solución glucosada al 33\% 200 c. c. por hora hasta completar 700 c. c. y luego 200 c. c. cada 8 horas (4). Nosotros, siguiendo la norma de recargar lo menos posible de substancias medicamentosas a las pacientes eclámpticas nos apartamos en mucho de lo aconsejado por la mayoría de los autores para lograr una rápida deshidratación.

Creemos que a un riñón que ha estado durante muchos días incapacitado por múltiples y variadas causas para desempeñar su función normal, es lo justo no violentarlo súbitamente, pudiendo conseguir lo que buscamos de manera inofensiva y razonable. Si a la acción de la azúcar sobre el asa de Henle, que es perjudi. cial (12), se' suma la sobrecarga de tener que eliminar no solamente los fluídos estancados sino también las grandes dosis administradas, debemos convenir que ese riñón, en un heroico esfuer. zo, sale casi siempre airoso, pero seguramente más lesionado de lo que se hallaba. ¿Cuántas de esas alteraciones renales que se 
consideran secuela de la toxemia habrán sido consecuencia de la medicación que se usó para forzarlo? A veces la respuesta del riñón no es tan afortunada sino que claudica, mostrándose incapaz para eliminar los edemas que había y los líquidos que de buena fe inyectamos. Muchas pacientes vimos fallecer por este motivo con edema agudo del pulmón cuando usábamos las isoluciones glucosadas hipertónicas.

Tampoco estamos de acuerdo con aquellos más cautos que aconsejan para las 24 horas 500 c. c. de líquido, fuera de los perdidos por orina, luego de la acción de 250 c. c. de glucosa al $50 \%$ (23). Si la intención es deshidratar, ¿ por qué se va a reintroducir el exceso de líquido que queremos sea eliminado?

Estando la eclámptica ahogada, ¿ para qué vamos a agregarle un centímetro cúbico de agua? Mientras el riñón no recupere su capacidad para eliminarla con facilidad, el trastorno general ten. drá que agravarse si contribuímos a aumentar la inundación.

Nosotros esperamos prudentemente a que comience la diuresis estimulada por la supresión de las causas que la estaban inhibiendo. Paralizados los núcleos grises hipotalámicos, y de hecho la secreción de adiuretina y vasopresina (4), por acción de la Acepromazina y el Fenobarbital; mejorada la irrigación renal en cancidad y calidad gracias al sulfato de magnesia y a la administración de oxígeno, el riñón, en esas condiciones favorables, comienza pronto a ejercer la anhelada función excretora.

Con el tratamiento medicamentoso expuesto atrás hemos controlado ya la eclampsia y la paciente se encuentra consciente entre las primeras tres horas siguientes a su aplicación. Adminisiramos entonces 500 miligramos a un gramo de Clorotiazida completando en esa forma la terapéutica deshidratante. La acción de la Clorotiazida se inicia a las dos horas de su ingestión y alcanza su mayor efecto 6 a 8 horas después. Aunque no se ha definido con exactitud el mecanismo por el cual ejerce su poderosa acción inhibidora sobre la reabsorción tubular del sodio, sí se ha demostrado en cambio que no causa daño alguno al riñón (15). Por eso creemos que nuestra conducta terapéutica contra la intoxicación hidrosalina ha llenado la finalidad buscada sin lesionarlo en lo más mínimo.

Transcurridas 12 horas desde el último ataque convulsivo, y ya la diuresis en plena actividad, permitimos desde entonces la ingestión de suero glucosado a voluntad de la paciente, suspendiendo aquí esa pequeña "cura de sed" a que la sometimos. 
Cuando entra en estado de aclampsia intercurrente, es decir, más allá de 24 horas sin ataques, suministramos una dieta pobre en cloruro de sodio durante tres días. Luego la consideramos como una preeclámptica controlada y la tratamos como tal.

Presentamos, después de las anteriores consideraciones, un resumen esquematizado del tratamiento en los distintos tipos cronológicos de eclampsia. Llamamos la atención sobre la forma de gestosis llamada "Inminencia de Eclampsia", caracterizada por la tríade signomática común a la preeclampsia más un grupo alarmante de síntomas, entre los que predominan la cefalea intensa, la epigastralgia y las alteraciones visuales, como manchas volantes, escotomas o amaurosis, además de una notoria excitabilidad. Todos estos síntomas son manifestaciones premonitorias de la eclampsia, aunque esté ausente bien sea la proteinuria o el edema aparente. El tratamiento de la Inminencia de Eclampsia es el mismo de la eclampsia anteparto o de la intraparto.

\section{Tratamiento de la Eclampsia Anteparto}

Se siguen las normas generales del tratamiento. Antes de verificar la exploración obtétrica aplicamos 15 c. c. de sulfato de magnesia al $15 \%$ por vía intravenosa lenta, seguido de 4 o 6 mi. ligramos de Acepromazina intramuscular. Luego que comprobamos que no se halla en trabajo de parto y que no hay alteración de los ruidos fetales, aplicamos 200 miligramos de Fenobarbital endovenoso, lentamente. Generalmente es suficiente este tratamiento. Si existe marcada excitabilidad repetimos media hora después la misma dosis de sulfato intravenoso y 200 miligramos de Fenobarbital intramuscular. Tres horas después, o tan pronto esté consciente la enferma, comenzamos a dar la Clorotiazida 500 miligramos cada 8 horas. Rara vez hemos tenido que repetir la aplicación de Acepromazina. El fenobarbital se sigue administrando por vía oral, 100 miligramos cada 8 horas.

En los 13 casos de eclampsia convulsiva ante-parto tratados en esa forma en el Servicio, seis horas después de su ingreso se encontraban todas las pacientes libres de ataques y completamente conscientes. A este grupo pertenecen las dos únicas muertes fetales de que hicimos mención atrás. Siete tuvieron parto con fetos vivos con peso menor de 2.500 gramos y las restantes parto con 
fetos vivos y peso superior a los 2.500 gramos. El mayor tiempo transcurrido en eclampsia intercurrente fue de 10 días y el menor de 36 horas. Se practicaron dos cesáreas: una por estrechez pélvica y otra por abruptio placentae con hemorragia que puso en peligro la vida de la madre.

\section{Tratamiento de la Eciampsia Intraparto}

En este tipo de eclampsia no solo interesa controlar las convulsiones, sino que importa conducir y atender inteligentemente el parto, pensando que es imperativo deber del obstetra velar en igual forma por la madre que por el hijo. Si dominamos medicamentosamente con éxito la toxemia y fracasamos en nuestra con. cucta como obstetras, tenemos que convenir en que no hicimos absolutamente nada. No quiere ello decir que ante el temor de que sucumba el feto tengamos que optar siempre por la conducta quirúrgica. No olvidemos lo que dice el español Fernández.Ruiz: "Si en obstetricia el parto no lo es todo, la cesárea tampoco lo es" (10).

De las normas generales tendremos muy en cuenta la necesidad de mantener libres las vías respiratorias y proporcionar una buena oxigenación durante todo el trabajo de parto. A pesar de que éste se hace casi siempre de manera muy rápida, es conveniente practicar apenas las exploraciones indispensables, ya que es posible que un tacto brusco o prolongado desencadene una nueva convulsión. Por eso somos partidarios de hacer el primer examen vaginal una vez que la acción medicamentosa ha comenzado su efiecto.

El tratamiento varía en algo al empleado para la eclampsia ante-parto. Pensando siempre en el feto no usamos los barbitúricos endovenosos de rutina, aunque la dosis que aconsejamos (200 miligramos) es prácticamente inofensiva. Aplicamos inicialmente 15 c. c. de sulfato intravenoso seguido de 4 miligramos de Acepromazina intramuscular. Si no hay signos de sufrimiento fetal y se repite el ataque aplicamos 100 miligramos de fenobarbital intravenoso. Si los hay repetimos el sulfato de magnesia intravenoso o aplicamos el barbitúrico intramuscular.

Así hemos tratado 10 eclampsias intraparto con todo éxito. Un solo feto pesó menos de 2.500 gramos y 5 sobrepasaron los 3.000 gramos. Se practicaron dos cesáreas por indicación obstétrica pura. (Cuadro número 2).

Detallamos aquí el caso número 5 ya que bien vale la pena reierirlo por cuanto refuerza el concepto de que la retención hidro- 


\section{ECLAMPSIAS AMTEPARTO}

\begin{tabular}{|c|c|c|c|c|c|c|c|c|c|c|c|}
\hline ORDEN & HISTORHA & $\begin{array}{l}\text { EQAD } \\
\text { Poria }\end{array}$ & $\begin{array}{l}\text { TENSIOM } \\
\text { ARTEPIAL }\end{array}$ & EDEAA & $\begin{array}{l}\text { Proteinu } \\
\text { ria }\end{array}$ & $\begin{array}{l}\text { FETO al } \\
\text { INGRESO }\end{array}$ & $\begin{array}{l}\text { NUMERO d' CON. } \\
\text { VULSFONES dESTE } \\
\text { de' TRATAMIEATO }\end{array}$ & PARTO & $\begin{array}{l}\text { ESTADO } \\
\text { DEL NINOO }\end{array}$ & PESO & $\begin{array}{l}\text { ESTANCIA } \\
\text { ontes del PACro }\end{array}$ \\
\hline 1 & 58793 & $2 t_{G_{4}} P_{3}$ & $145 / 110$ & III & 1.26 & Vivo & una & espont. & moerto & $800_{8}$ & 4 dias \\
\hline 2 & 60208 & ${ }^{20} G, P_{0}$ & $165 / 120$ & $I$ & 7.77 & vilo & nirnguna & espont. & VIVo & 1620 & 2 dias \\
\hline 3 & 60076 & $20, P_{B}$ & $135 / 90$ & $I I$ & 0.21 & Vivo & ninguna & Inouccions & vivo & 2900 & 4 días \\
\hline 4 & 52999 & ${ }^{18}, P_{0}$ & $160 / 90$ & $\Leftrightarrow$ & 6.72 & Vivo & una & espont & Viro & 1500 & 2 dias \\
\hline 5 & 59682 & ${ }^{18} \mathrm{gip}$ & $130 / 80$ & $I$ & 4.86 & ivo & ninguna & espont & Vivo & 2360 & $1 / 20165$ \\
\hline 6 & 55 & $22, P$ & $135 / 90$ & $\Leftrightarrow$ & 2.73 & Vivo & ninouna & cesarea & viro & 3100 & 6 dias \\
\hline 7 & 58 & ${ }^{34} Q_{1} P_{0}$ & $140 / 90$ & II & 7.35 & Viro & ninguna & espont & VIVO & 2500 & 19 dies \\
\hline 8 & 22 & $20_{G 2} P$ & $190 / 120$ & $(-)$ & 5.90 & Vivo & una & espont. & muerto & 1360 & 19 dias \\
\hline 9 & 58934 & ${ }^{19}, P_{0}$ & $130 / 90$ & 1 & 0.21 & VIVO & ninguna & espont & Viro & 2480 & 6 dias \\
\hline 10 & 55708 & $356 P_{3}$ & $100 / 60$ & $I$ & 2.68 & muerto & minguna & cesarea & muerto & 1300 & 16 horas \\
\hline 11 & 58636 & $20, P_{0}$ & $145 / 90$ & $I$ & 0.21 & KIVO & zind & forceps & Vivo & 3560 & 2 diass \\
\hline$\angle 2$ & 54007 & $22, P$ & $140 / 120$ & II & 0.52 & Vivo & ninouna & espont. & vivo & 2400 & 13 dias \\
\hline 13 & 61319 & $176, p_{0}$ & $130 / 110$ & II & 4.20 & Vivo & 205 & forceps & vivo & 2000 & 2 dios \\
\hline
\end{tabular}

- Cesarea por estrechéz petivica

* Abruptio placeniae

* Cesarea previa. Abruptio placenta con hemorragia que puso en peligna la vida de la madre. 
salina juega papel de extremada importancia en la génesis de la eclampsia. Se trataba de una paciente de veinte años con el siguiente diagnóstico de ingreso: primigestante con 40 semanas de smbarazo, feto vivo, estrechez pélvica eclampsia intraparto. Ten. sión arterial 190/110, 1.47 gramos de proteinuria, edema generalizado. Se aplicó el tratamiento repitiendo un ataque convulsivo. Cinco horas después de su ingreso se practicó cesárea bajo anestesia raquídea, obteniéndose un niño vivo de 3.400 gramos. En las prineras 12 horas de post-operatorio la paciente se hallaba inconsciente, en anuria y había presentado cinco ataques corvulsivos. Aplicamos. a manera de terapéutica heroica, 2 c. c. de un diurético mercurial. Dos horas más tarde se iniciaba la diuresis, dosapareciendo desde entonces las manifestaciones convulsivas. Diez días después salían del servicio en buenas condiciones madro e hijo.

Recomendamos en la conducción y atención del parto dos hechos importantes: ei primero es la amniotomía artificial en el momento del primer tacto, si la presentación apoya sobre el cuello, sin importar el grado de dilatación. Dos beneficios obtenemos con ella: evitamos el abruptio placentae y acortamos el periodo dilatante. La segunda recomendación es la aplicación sistemática de fórceps bajo o de desprendimiento, utilizando el bloqueo pudendo como anestesia. No hay que olvidar que los agentes anestésicos inhalados están formalmente contraindicados y que la anestesia raquídea no es aconsejable (23).

Una vez verificado el alumbramiento aplicamos ergotrate in. tramuscular.

\section{Tratamiento de la Eclampsia del puerperio}

Es la forma menos frecuente cie eclampsia. Aseguramos que áesaparecerá si el obstetra siempre tiene el cuidado de aplicar harbitúricos en los primeros minutos que siguen a la expulsión del feto en toda paciente que haya presentado preeclampsia-eclampsia, repitiendo la dosis cada 8 horas durante las primeras 24 o 36 horas de puerperio.

Los cuatro casos de eclampsia del post-parto que hemos tratado, tres han sido pacientes que tuvieron su parto fuera del Servicio y la otra una paciente preeclámptica a quien no se aplicó medicación anticonvulsivante después del parto. 
Cuadro № 2

\section{ECLAMPSIAS INTRAPARTO}

\begin{tabular}{|c|c|c|c|c|c|c|c|c|c|c|c|c|}
\hline OROEA & HISTORIA & EDAD & PARIDAD & $\begin{array}{l}\text { TENSION } \\
\text { ARTERIAL }\end{array}$ & EDFHA & $8^{60^{6 t}}$ & $\begin{array}{l}\text { FETO a/ } \\
\text { INGRESO }\end{array}$ & $\begin{array}{l}\text { Ne de cowrut swores } \\
\text { okspues del treat. }\end{array}$ & PARTO & $\begin{array}{c}\text { ESTADO } \\
\text { NIETO } \\
\text { NINO }\end{array}$ & PESO & $\begin{array}{l}\text { ESTANCHA } \\
\text { entes del perto }\end{array}$ \\
\hline 1 & 57781 & 22 & $\overline{G_{1} P_{0}}$ & $150 / 110$ & 71 & 6.30 & vivo & una & esp. & vivo & 2800 & 12 horas \\
\hline 2 & 58562 & 30 & $G_{3} P_{2}$ & $170 / 120$ & II & 0.63 & muerto & ningund & esp. & reverto & 1620 & 28 horas \\
\hline 3 & 58526 & 19 & $G, P_{0}$ & $180 / 130$ & 2 & 2.73 & viro & ninguna & fore. & Vivo & 2840 & 1 hora \\
\hline 4 & 58157 & 24 & $G, P_{0}$ & $170 / 120$ & III & 1.26 & Vivo & ninguna & cesat & VIVO & 2500 & 4 horas \\
\hline 5 & 59488 & 20 & $G, P_{0}$ & $190 / 110$ & III & 1.47 & vivo & seis & cesay & Vivo & 3400 & 5 horas \\
\hline 6 & 59344 & 25 & $G, P_{\Delta}$ & $220 / 120$ & $(-)$ & 1.89 & viro & ninguna & fore & vivo & 3260 & 5 hotas \\
\hline 7 & 59322 & 32 & $G g_{9} P_{8}$ & $140 / 100$ & $I$ & 10.50 & Vivo & ningund & esp. & Vivo & 1800 & 2 horas \\
\hline 8 & 59942 & 18 & GiPo & $200 / 130$ & II & 5.25 & vivo & ninguna & esp. & Vivo & 3660 & 5 horas \\
\hline 9 & 60027 & 16 & $G . P_{0}$ & $140 / 90$ & $I I$ & 0.21 & vivo & Una & fore & rivo & 3200 & 4 horas \\
\hline 10 & 68861 & 25 & $G_{1} P_{0}$ & $160 / 80$ & I & 2.05 & Vivo & ninguna & esp. & Vivo & 3100 & 3 horas \\
\hline
\end{tabular}

- Cesarea par sufrimiento fetal. Falta de encajamiento (presentación 0.5.)

* Cesanca par estrachei pelvica. Un atague antes de la intarrención. Tratamiento insufieiente. Cinco atopues en el puetpeno. 


\section{ECLAMPSIA POST_PARTO}

\begin{tabular}{|c|c|c|c|c|c|c|c|}
\hline Orden & Historia & Edad & Paridad & Edema & Proteinuria & $\begin{array}{l}\text { Tensión } \\
\text { arterial }\end{array}$ & $\begin{array}{l}\text { Ataques } \\
\text { después } \\
\text { del trat. }\end{array}$ \\
\hline 1 & $y 439$ & 23 & $\mathrm{G}^{3} \mathrm{P} 1$ & $(-)$ & 0.21 & $90 / 50$ & ninguno \\
\hline 2 & 59635 & 19 & G1P1 & $(-)$ & 0.73 & $85 / 50$ & ninguno \\
\hline 3 & 59282 & 27 & G10P8 & II & 2.10 & $160 / 120$ & ninguno \\
\hline 4 & 57788 & 21 & $\mathrm{G}^{1} \mathrm{P}^{1}$ & I & 5.46 & $170 / 120$ & ninguno \\
\hline
\end{tabular}

Este tipo de eclampsia la tratamos aplicando 200 miligramos de Fenoborbital intravenoso y 4 miligramos de Acepromazina intramuscular. Luego 100 miligramos de Fenobarbital cada 8 horas por via oral.

\section{Conclusiones}

Se ha expuesto un sistema o método de tratamiento para la preeclampsia-eclampsia utilizado durante cuatro meses en el Servicio de Toxemias del Instituto Materno-Infantil "Concepción Villaveces de Acosta", sustentado en bases fisiopatológicas, comparado con los anteriormente usados y demostrada estadísticamente su bondad.

\section{BIBLIOGRAFIA}

1. ALVAREZ H., CALDEYRO-BARCIA R.- "Fisiopatología de la contracción uterina y sus aplicaciones en Clínica Obstétrica”. Relato oficial presentaco al II Congreso Latinoamericano de Obstetricia y Ginccología. Páginas 74,75 .

2. ASSALI N. S.-“Treatment of Eclampsia and Preeclampsia". Cl. Obst. and Gyn. Vol. 1 № 2. June 1958.

3. BOtella LlUSiA J.-“Endocrinología de la mujer”. $2^{\text {a }}$ edición. Ed. Cient. Méd. 1956. Páginas 123, 727, 729.

4. BOTELLA LLUSIA J.-"Patología Obstétrica". Ed. Cient. Méd. Barcelona. 1955. Páginass 80, 82.

5. CASTAÑO CASTILLO C.- “El Plegici!, un neuropléjico de confianza". Trabajo original ^ inédito para publicar en el Boletín Científico de la Caja Nacional de Previsión.

6. CLIN-COMAR Laboratoires.-"Plegicil. Neurcpléjico de gran actividad 3. pequeñas dosis".

7. DELAY J., PICHOT P., ROBERT R.-“Un nuevo medicamento neuro'éptico en siquiatría". Extracto de "La Presse Medicale". № 22. Mayo 16 de 1957.

8. DEXEUS-FONT S.-Progresos de Gin. y Obst. Vol. I. Fasc. 2. 1958. Página 342 . 
9. ELI LILLY and Company.-“Terapéutica con los Barbitúricos”. Publicación de Lilly.

10. FERnANDEZ-RUIZ C.--"El Camino del Ginecólogo”. Ed. Cient. Méd. Madrid, 1956. Página 24.

11. FERNANDEZ BASTIDAS M. A.-“Toxemias gravídicas". ICSS. Clínica 19 de Mayo. Bogotá. 1955. Página 19.

12. GOODMAN L., GILNAN A.-"Bases Farmacológicas de la Terapéutica”. Ed. Uteha. México. 1945. Páginas 140, 170, 211.

13. HENAO-CABAL O., MUÑOZ DELGADO S.-"Patología placentaria de İ. Toxemia del embarazo". Re. Col. de Obs. y Gin. Vol. № 2. Marzo, abril 1958. Página 122.

14. LEON J.-“Tratado de Obstetricia”. Ed. Cien. Arg. $1^{\text {a }}$ edición. 1957. Tomo II. Páginas 320, 449.

15. MERCK SHARP \& Dohme International.-“"El edema y los diuréticos en la práctica médica".

16. MORARI M.- "Inhibición de la lactación por sedación del diencéfalo". Prog. de Obst. y Gin. Vol. I. Fasc. I. 1958. Página 99.

17. MOODY M. C.-“Preeclampsia y Eclampsia”. Rev. Mundo Médico. Vol. V. No 2. Feb. marzo. 1958. Página 75.

18. MORRIS N.- "Tratamiento de la toxemia gravídica con medicamentos hipotensores". Rev. Triángulo. Vol. II. No 8. Marzo. 1957. Página 327.

19. MUNOZ DELGADO S., HENAO-CABAL O.- "Patología de la Toxemia ciel embarazo en madres e hijos". Rev. Col. de Obst. y Ginec. Vol. IX. N ${ }^{\circ} 2$. Mayo, abril, 1958.

20. RUZICSKA J., ZSOLNAI B.-"Sobre el tratamiento de las toxemias del embarazo con Largactil-Fenergán". Progr. de Obst. y Ginec. Vol. I. Fasc. 2. 1958. Página 341.

21. SANCHEZ LOPEZ A.- "Tratamiento de la Eclampsia puerperal mediante neuropléjicos". Toko-Ginecología Práctica. Año XIII. N 122. Mayo, 1954.

22. SILVA MOJICA C. R., RAMIREZ SANCHEZ J., GOMEZ P. J. A."Tratamiento de la Toxemia gravídica". Rev. de la Fac. de Med. U. N. Vol. 26. Númercs 7, 8. Julio, agosto. 1958.

23. TATUM H. J.-“Toxemias del embarazo". "Método de Tatum". Traducción mineográfica de la Fac. de Nied. U. del Valle, Cali.

24. TATUM H. J.-“Compartmental Distribution and Shift of Water and Electrolytes in Pre-eclampsia”. Am. Jorn. of Obs. and Gyn. Vol. 67. N $N^{\circ}$ 6. Páginas 1.197, 1.201. June 1954.

25. WILKINS R. W.- "Precauciones necesarias para el uso de las drogas antihipertensivas incluyendo Clorotiazida". The Journ. of the Amer. Med. Assoc, vol. 167. No 7 June 14, 1958. 